\title{
Gravitational waveswaves on Sun?
}

\author{
The notion that a solar oscillation may be stimulated by a train of gravitational waves from a nearby \\ binary star, however preliminary, is an intriguing pointer to novel methods.
}

A BOMBSHELL was hidden among the more political announcements of the directorgeneral of the French Centre National de la Recherche Scientifique (CNRS) last week - a claim that French astrophysicists, in conjunction with Italian and British colleagues, had detected gravitational waves impinging on the Sun. The waves, the group said, are coming from the gamma and X-ray source Geminga, and can be detected in the form of a solar oscillation.

The evening newspaper Le Monde, given early warning of the announcement, made it a front-page lead on 11 October with the headline "A major discovery in astronomy - confirming a theory of Einstein", while earlier that day Philippe Delache, the prime mover of the group, was holding up a proof of the front page to a European Space Agency committee meeting at Frascati near Rome. The committee was deciding between various proposals for new scientific satellites, among them SOHO - one of whose missions is to measure solar oscillations. SOHO was one of the satellites ultimately selected, although this may have had nothing to do with Delache's presentation (see p.660).

But, said Delache later, "we must be very, very cautious" in the interpretation of the data. His view was echoed by the British partner in the potential discovery, George Isaak of the University of Birmingham.

The tale, so far unpublished, is this. At two scientific meetings during the summer, Isaak had raised an interesting possibility: that the Sun might be used as a detector of gravitational waves. First, its mass (which determines coupling to gravity) is obviously much larger than any experimental apparatus on Earth. And second, although this mass is concentrated at the centre (where the density is $100,000 \mathrm{~kg} \mathrm{~m}^{-3}$ ) a gravitational wave which shook the centre could propagate out to the Sun's surface and be detected there as a solar oscillation. Moreover, if it propagated as a pressure wave (a sound wave), its amplitude would grow as it rose into the more rarefied layers of the Sun, amplifying by perhaps a factor of 100,000 from core to surface "like the cracking of a whip".

Then, "in a flippant mood", as Isaak describes it, he suggested that the solar 160-minute mode - normally regarded as far too long to be a pressure wave but a mode whose amplitude is difficult to explain on any basis - might in fact be driven by the absorption of a gravitational wave at that frequency. Delache (of the Observatoire de Nice) heard the suggestion, and set to work with Jacques Paul (CEA, Saclay) and G. F. Bignami (CNR, Milan) to see if there was any sign of a 160-minute mode in the gamma radiation of Geminga, whose radiation had previously been found to be steady.

Why Geminga? Because the steepness of its X-ray spectrum - indicating little interstellar absorption - in combination with a very low visual magnitude (21.2 according to an identification by the CanadaFrance-Hawaii telescope) suggests that Geminga might be the closest condensed object to our Solar System. Then, if it were a binary condensed system, and if it had a frequency of 160 minutes, it could be radiating gravitational waves that could drive the Sun's 160-minute mode.

So Delache and company set out to make a fast Fourier transform of five months' gamma observations, spread over seven years, of Geminga from the satellite COSB. And they found, they say, a 160 -minute period. Taking two months' data alone, the 160-minute signal is detectable, says Delache, and it increases in amplitude as 3, 4 and 5 months' data are taken. Background radiation at the same intensity showed no 160-minute signature.

Moreover, the Geminga period does not exactly match the period of the solar oscillation - it is exactly one cycle per (Earth) year different. This would be explicable if the mode stimulated in the Sun has a non-zero azimuthal number $m$, or in other words, if it travels around the solar equator. For then an Earth-bound observer would see one cycle more (or less, according to the direction of travel of the wave) each year than a stationary observer. Since we do not travel around Geminga, however, we see that at its true frequency. That $m$ of the 160-minute mode should be non-zero then becomes a testable prediction of the gravitational wave hypothesis.

So far, so good - but there are difficulties. If the 160 -minute mode is not a pressure wave, Isaak's amplification argument does not apply, as he himself points out. "There is a problem with the amplitude", Delache concurs, at both ends of the radiation: at Geminga and at the Sun. The power of a binary gravitational radiation source (the radiation comes from the advance and retreat of the two masses) varies as the fifth power of the mass, says Delache, but Geminga's mass is unknown. And at the Sun, the dynamics of the 160-minute mode are not understood.

Furthermore, a British solar theorist pointed out last week that the gravitational radiation from a binary is emitted predominantly at twice the orbital frequency, whereas the gamma radiation would vary at the orbital frequency, unless our line of sight lay in the plane of the orbit (possible, but unlikely). Delache is undaunted by this argument, however: perhaps each of the condensed partners has gamma-emitting hot-spots, he suggests.

Delache does point out, however, that there is some evidence of a 160 -minute period in microseismicity of the Earth which could be explained by the further influence of Geminga's gravitational radiation on the Earth. Much now seems to depend on the theorists, and the calculation of amplitudes; but meanwhile Delache and colleagues are going on to analyse the $\mathrm{X}$-ray radiation from Geminga as detected by the Einstein Observatory.

More news about gravitational waves has come this week from Eduardo Amaldi and his group in Rome, who are seeking gravitational waves with the now more traditional suspended bars of metal. They were the reluctant source of Italian headlines claiming a source of gravitational radiation at the centre of the Galaxy. "We didn't want to publish anything", Amaldi said this week. Amaldi and his group have been studying background in their detector - single bar spikes - " "and we've found some periodicity; but they're rather large signals and we don't believe they are gravitational waves", Amaldi said.

However, "one of the periods" is identical with the sidereal day (the true period of rotation of the Earth relative to the fixed stars), and the maximum of the cycle is "not far, within rather large errors" from the direction of the centre of the Galaxy.

And strangely enough, the very first claimant of the discovery of gravitational waves - Joe Weber of the University of Maryland - also believed his waves were coming from the galactic centre. Could it be, perhaps, that there is a galactic source, and that it is not the detector itself but the Earth that is reacting to the waves, transmitting the effect through seismicity? It is difficult to say until the work is published.

So when will Amaldi publish? "It's a long business' he said. "We are very very careful. These are very small effects." But with large consequences, one might add.

Robert Walgate 